Cahiers $d u$ MONDE RUSSE

\section{Cahiers du monde russe}

Russie - Empire russe - Union soviétique et États indépendants

61/3-4 | 2020

Écritures visuelles, sonores et textuelles de la justice

\title{
Andy BYFORD, Science of the Child in Late Imperial and Early Soviet Russia
}

\section{Cécile Pichon-Bonin}

\section{OpenEdition}

\section{Journals}

Édition électronique

URL : https://journals.openedition.org/monderusse/12144

DOI : $10.4000 /$ monderusse. 12144

ISSN : 1777-5388

\section{Éditeur}

Éditions de l'EHESS

\section{Édition imprimée}

Date de publication : 1 juillet 2020

Pagination : $528-530$

ISBN : 978-2-7132-2832-2

ISSN : $1252-6576$

Référence électronique

Cécile Pichon-Bonin, "Andy BYFORD, Science of the Child in Late Imperial and Early Soviet Russia ", Cahiers du monde russe [En ligne], 61/3-4 | 2020, mis en ligne le 01 juillet 2020, consulté le 14 septembre 2022. URL : http://journals.openedition.org/monderusse/12144 ; DOI : https://doi.org/ 10.4000/monderusse. 12144 


$\begin{array}{llllllllllllllllllllllllll}\mathrm{F} & \mathrm{I} & \mathrm{N} & & \mathrm{D} & \mathrm{E} & & \mathrm{L} & & \mathrm{A} & \mathrm{N} & \mathrm{C} & \mathrm{I} & \mathrm{E} & \mathrm{N} & & \mathrm{R} & \mathrm{E} & \mathrm{G} & \mathrm{I} & \mathrm{M} & \mathrm{E} \\ \mathrm{E} & \mathrm{T} & & \mathrm{D} & \mathrm{E} & \mathrm{B} & \mathrm{U} & \mathrm{T} & & \mathrm{D} & \mathrm{E} & & \mathrm{L} & \mathrm{A} & & \mathrm{P} & \mathrm{E} & \mathrm{R} & \mathrm{I} & \mathrm{O} & \mathrm{D} & \mathrm{E}\end{array}$

Andy BYFORD

Science of the Child in Late Imperial and Early Soviet Russia

Oxford : Oxford University Press, 2020, 312 p.

Andy Byford offre une histoire inédite et ambitieuse de la science de l'enfant, en Russie et en URSS, des années 1880 aux années 1930. S'intéressant au développement et à la socialisation de l'enfant, la science de l'enfant est ici décrite comme un mouvement fondamentalement interdisciplinaire et interprofessionnel. Impliquant enseignants, psychologues, médecins, parents, juristes et administratifs, elle convoque des notions, des méthodes et des pratiques relevant de la médecine, de l'éducation spécialisée, de la psychologie du développement et de l'éducation, de la psychiatrie enfantine, de la criminologie adolescente et de l'anthropologie de l'enfance. Elle aborde l'enfant comme un objet de recherche scientifique, d'expertise professionnelle, d'intérêt et de soins publics, d'action sociale. Cette simple présentation permet déjà de saisir l'ampleur des recherches effectuées sur une grande diversité d'acteurs, de domaines et d'institutions.

Sur la base de cette définition, l'auteur insiste sur l'importance de ne pas considérer la science de l'enfant comme un objet et sur la pertinence de la dé-réifier afin de l'étudier en tant qu'espace de mobilisation et d'interactions de dynamiques disciplinaires et professionnelles. Il examine ainsi cette dimension, jusqu'alors idéalisée ou critiquée, mais jamais analysée en soi, comme il le rappelle. Le livre explique très clairement la façon dont se structure, s'institutionnalise et fonctionne ce champ très hétérogène, mêlant travail scientifique et pratiques professionnelles, connaissances et expertises. Il expose les différentes idées et les concepts issus des divers domaines théoriques et analyse la récupération, l'encadrement, l'organisation, l'instrumentalisation qui mènent à la constitution de la pédologie comme « science d'État ». Prenant un recul salutaire, Andy Byford considère les différentes définitions du terme pédologie et analyse ses usages. Il innove en rendant apparentes ses diverses fonctions de mobilisation de groupes professionnels spécifiques, de moteur et de cadre d'intégration, de programme de contrôle et de pratique professionnelle et en prenant en compte leurs complexes imbrications. En saisissant la science de l'enfant comme phénomène historique, l'auteur atteint ses objectifs : l'ouvrage contribue simultanément à une meilleure connaissance des différents aspects de la science de l'enfant et participe à l'écriture de l'histoire des sciences humaines russes et soviétiques, des professions, de l'éducation et de l'enfance, de l'intérêt scientifique pour le développement biopsychosocial de l'enfant.

Après une préface retraçant le cheminement intellectuel de l'auteur, s'ouvre une introduction précisant les origines culturelles, sociales et politiques, et les étapes de la constitution d'une science de l'enfant en tant que mouvement, compris dans une double perspective russe et transnationale. Le livre se compose de six chapitres 
progressant selon un ordre chronologique et thématique. Le premier présente le contexte social et scientifique dans lequel l'étude de l'enfance s'est constituée, du milieu du $\mathrm{XIX}^{\mathrm{e}}$ siècle au début du $\mathrm{XX}^{\mathrm{e}}$ siècle. Le deuxième chapitre retrace l'institutionnalisation du mouvement de la science de l'enfant des années 1890 à 1917. Il traite des pratiques scientifiques qui se sont cristallisées autour de l'éducation scolaire. Le troisième chapitre est le dernier consacré à l'époque impériale, il examine le rôle des diagnostics et du traitement de différentes « déficiences » de la population enfantine. Vient ensuite une partie sur l'expansion des institutions dédiées à l'étude de l'enfant durant la guerre civile et au début des années 1920, puis un cinquième chapitre consacré à l'institutionnalisation de la pédologie comme « science d'État ». Celui-ci se concentre sur l'évolution des formes et des significations de la pédologie soviétique au cours des années 1920 et insiste sur le tournant des années 1920-1930. Le dernier chapitre est dédié à la pédologie comme domaine d'activité au sein du système scolaire qui se met en place à partir de ces années charnières.

La dé-réification de la science de l'enfant permet d'aborder sous un angle nouveau différentes questions traversant l'historiographie consacrée à la Russie et à l'URSS. Sans épuiser le riche contenu de l'ouvrage, citons ici quelques exemples. La question des causes et des conséquences de la condamnation de la pédologie par le Parti communiste en 1936 est ainsi revisitée. Andy Byford explique que ce rejet concerne davantage la pédologie comme pratique professionnelle que comme science en tant que telle. Sa vision de la pédologie comme champ de force complexe lui permet de sortir d'un paradigme fréquent de l'histoire soviétique qui consiste à réhabiliter un objet d'étude condamné à la période stalinienne, en construisant un contre-argumentaire terme à terme.

Tout en exposant les idées et concepts de l'époque, Andy Byford explique de façon convaincante que les tensions et rivalités traversant le champ de la science de l'enfant proviennent moins de désaccords théoriques sur les notions relatives au développement et à la socialisation de l'enfant qu'à la façon dont chacun aborde la constitution même de la pédologie en tant que domaine scientifique. Il s'agit donc moins d'enjeux de contenus de cette science que de la manière dont on définit la discipline, son identité, et dont on pense sa place et son rôle. À nouveau, l'auteur propose un regard décentré et aborde de manière nouvelle les débats des années 1920 et du tournant des années 1920-1930, souvent résumés à une opposition binaire entre les approches biogénétique et sociogénétique au sein de la théorisation du développement.

La prise en compte des acteurs, des institutions, des discours et des pratiques dans leurs interactions construit une histoire riche de l'effort de synthèse qui préside à la constitution de la pédologie en science et de la centralisation institutionnelle qui s'organise à la fin des années 1920 et au début des années 1930. La construction de la pédologie en "science d'État » est analysée dans ses étapes et ses finalités. Le lecteur est invité à envisager sous différents angles les rapports entre théorie et pratique, une autre problématique récurrente dans l'historiographie soviétique. Il suit l'utilisation de cette science pour réduire le fossé séparant les idéaux de la 
réalité, le passage d'une théorie convoquée pour légitimer les nouvelles politiques pédagogiques à une théorie toujours plus impliquée dans l'élaboration même des réformes. La description de cette évolution apporte des éléments nouveaux et nourrit les réflexions antérieures sur les ruptures, les continuités et les inflexions entre les différentes périodes définies par le découpage historique. En particulier entre l'époque tsariste et l'ère soviétique, puis entre les années 1920 et le début du stalinisme. L'auteur revient notamment sur le déroulement des campagnes de dénonciation des années 1929-1932 dans le domaine de l'éducation, envisageant la réorganisation de la pédologie en tant que science au service de l'école, comme une façon de reconnaître les « erreurs » commises et de sauver le champ d'une possible disparition. En observant les stratégies à l'œuvre, il restaure ainsi la complexité d'une situation souvent placée sous l'égide d'une mise au pas des différents champs scientifiques et culturels par le Parti.

Andy Byford parvient à rendre son discours novateur et érudit accessible aux étudiants et chercheurs, mais aussi aux personnes curieuses du domaine et intéressées par l'histoire de l'enfance et de l'éducation. Il croise des sources particulièrement nombreuses et variées, fondant ainsi son analyse sur un matériau abondant. Il propose, en parallèle du texte, un appareil de notes fourni. Le seul regret du lecteur historien peut porter sur le choix éditorial de faire figurer en fin d'ouvrage une bibliographie recensant uniquement les sources secondaires et renvoyant aux notes de bas de page pour les sources primaires, publiées et archivistiques. Un paragraphe introductif présentant les sources et explicitant leur traitement aurait également été bienvenu pour compléter la préface qui prend partiellement en charge cet aspect. Cette remarque ne saurait bien entendu remettre en cause la grande qualité d'un livre dans lequel l'érudition sert le renouvellement des cadres interprétatifs de l'histoire russe et soviétique. Andy Byford signe ici un ouvrage de référence sur le sujet.

Cécile Pichon-Bonin

CNRS, LIR3S 